\title{
Aspectos históricos e constitucionais da taxa de incêndio do estado do Rio de Janeiro
}

Historical and constitutional aspects of the tax of fire prevention and fire extinction of Rio de Janeiro state

Aspectos históricos y constitucionales de la tasa de incendios del estado de Río de Janeiro

Revista Dissertar No32 V.1 ANO XV

DOI: $10.24119 / 16760867$ ed115257

Data de submissão: 29-11-2018

Data de aceite: 01-05-2019

por Heitor Gama Pimentel ${ }^{1}$

\section{Resumo}

O presente artigo objetiva analisar, sob o enfoque históricoconstitucional, o tributo denominado taxa de prevenção e extinção de incêndios instituída pelo Estado do Rio de Janeiro. Almeja aferir a conformidade da referida exação com o texto constitucional, bem como investigar, neste ponto, à luz da jurisprudência do Supremo Tribunal Federal, quais são os critérios adotados pela Suprema Corte para a verificação da constitucionalidade das taxas de serviços públicos instituídas no âmbito dos Estados da Federação.

Palavras-chave: Direito Constitucional; Sistema Tributário Nacional; Taxas de Incêndio; Constitucionalidade

\section{Abstract}

The present article aims to analyze, under the historical and constitutional aspect, the tax of fire prevention and fire extinction required by the State of Rio de Janeiro. The proposal is the review of this fee accordance with the Constitution, as well as investigation, in the light of jurisprudence of Supreme Court, what are the rules adopted to the public service tax creation by the States of the Federation.

Keywords: Constitutional Law; National Tax System; Tax of Fire Prevention and Fire Extinction; Constitutionality

1 Heitor Gama Pimentel: Especialista em Direito Público pela Universidade Cândido Mendes/RJ. Advogado e Professor Universitário (UNESA/ RJ). Membro do Núcleo Observatório Ontológico de Pesquisa - NOOP da UNESA/Petrópolis. heitorgamapimentel@gmail.com 
144 Aspectos históricos e constitucionais da taxa de incêndio do estado do Rio de Janeiro

\section{Resumen}

El presente artículo tiene por objeto analizar, bajo el aspecto históricoconstitucional, el tributo denominado tasa de prevención y extinción de incendios instituida por el Estado de Río de Janeiro. En este punto, a la luz de la jurisprudencia del Supremo Tribunal Federal, cuáles son los criterios adoptados por la Corte Suprema para la verificación de la constitucionalidad de las tasas de servicios públicos instituidas en el marco de los procedimientos de los Estados de la Federación.

Palabras clave: Derecho Constitucional; Sistema Tributario Nacional; Tasas de Incendio; Constitucional

\section{Introdução}

O estudo ora colimado objetiva apreciar a questão da constitucionalidade da taxa de serviços Estaduais, relativa à Prevenção e Extinção de Incêndio, popularmente conhecida como taxa de incêndio, cobrada pelo Corpo de Bombeiros Militar do Estado do Rio de Janeiro no âmbito de suas atribuições fiscais. Desde a sua criação até os presentes dias, o referido tributo tem sido alvo de constantes surtidas por parte dos contribuintes, os quais, em sua maioria, discordam da referida exigência.

No âmbito da evolução jurisprudencial do Supremo Tribunal Federal, a questão das taxas de prevenção e combate ou extinção de incêndios retornam à pauta de maneira recorrente, tendo em vista que aquele Tribunal possui entendimento sedimentado de que as taxas de serviços públicos se prestam tão somente a remunerar serviços públicos específicos e divisíveis. Seriam as taxas de incêndio, em conflito com o disposto no art. 142, inciso II da Constituição da República, tributo destinado a remunerar serviços públicos universais, o que seria papel dos impostos.

Desta forma, servirá o presente trabalho para o desenvolvimento dos aspectos conceituais e da abordagem histórica acerca da taxa de incêndio e seu posterior cotejo frente à literatura especializada e também aos julgados do Supremo Tribunal Federal nos últimos anos a respeito das taxas de serviços públicos e sobre quais fatos incidem.

O estudo que ora se propõe é analítico e exploratório, e terá como base a evolução da jurisprudência da Suprema Corte brasileira e também a opinião ligada à doutrina já consagrada sobre o tema.

\section{As taxas de serviços públicos: aspectos conceituais}

São cinco as espécies de tributos previstas na Constituição da República e chanceladas pela jurisprudência do Supremo Tribunal Federal : (i) impostos, (ii) taxas, (iii) contribuições de melhoria, (iv) 
empréstimos compulsórios e (v) contribuições especiais. Para este estudo, duas dessas espécies possuem maior relevância: os impostos e as taxas.

Os impostos são tributos destinados a remunerar serviços públicos universais, indivisíveis, ou seja, aqueles serviços prestados à toda comunidade; um número indeterminado de pessoas, sem qualquer individualização (FALCÃO, 1999, p. 77). Isto ocorre porque determinados serviços, tratados pelo Poder Constituinte Originário como universais, devem ser prestados à população independentemente da capacidade individual dos cidadãos de pagar por tais serviços (ROSA JUNIOR, 2009, p. 62-63). Esta foi uma opção do Poder Constituinte no que tange aos serviços universais, tais como saúde pública, segurança pública, iluminação pública, arquivos públicos et cetera (ARAGÃO, 2012, p. 374).

É também por este motivo que os impostos são tributos de caráter contributivo - não retributivo, - onde suas receitas se destinam a fazer caixa e são de livre aplicação, sem vincular-se a qualquer atividade ou gasto específico do Estado, em regra (CARRAZZA, 2004, p. 475; FALCAO, 1999, p. 77). O objetivo foi conferir ao Administrador maior liberdade na alocação de recursos durante a atividade de governança, principalmente no que tange ao manejo de gastos para a manutenção dos serviços universais essenciais (ROSA JUNIOR, 2009, p. 62-63).

Ademais, são os impostos tributos cobrados independentemente de qualquer contraprestação estatal específica, pois não há necessidade que, em relação ao contribuinte, individualmente tratado, surja a prestação direta do serviço (CARRAZZA, 2004, p. 474-475).

As taxas, por sua vez, podem ser subdivididas, segundo o disposto no artigo 145, inciso II da Constituição, em taxas de polícia, consistentes basicamente nas taxas ligadas aos serviços de fiscalização - tais como fiscalização ambiental, sanitária e de posturas - ou em taxas de serviços públicos (BALEEIRO, 2005, p. 540-541), sendo estas últimas as principais financiadoras dos serviços públicos específicos não universais, tais como coleta e remoção domiciliar de lixo e emissão de determinados documentos.

Por expressa previsão constitucional, as taxas de serviços públicos apenas podem ser exigidas no caso de prestação de serviços específicos e divisíveis, prestados ao contribuinte ou postos à sua disposição, e nunca devem se valer a remunerar serviços universais, prestados à toda coletividade, sem individualização. Isso denota a natureza retributiva das taxas, oposta ao caráter contributivo e mais amplo dos Impostos (ROSA JUNIOR, 2009, p. 81).

Dá-se que diversas são as situações onde o Poder Público, em afronta direta aos ditames constitucionais, institui taxas com o fito de 
146 Aspectos históricos e constitucionais da taxa de incêndio do estado do Rio de Janeiro

remunerar serviços públicos universais.

\section{O entendimento do Supremo Tribunal Federal sobre as taxas de iluminação pública}

Durante vários anos, diversos Municípios instituíram eexigiram as chamadas taxas de iluminação pública (TIP). A referida taxa se prestava a remunerar os serviços de iluminação pública fornecidos pelos Municípios, contudo, a doutrina especializada sempre condenou o uso das taxas para tal finalidade, tendo em vista que a iluminação pública é, notadamente, um serviço público de caráter universal e indivisível, e que só pode, portanto, ser remunerado através das receitas dos impostos (TORRES, 1999, p. 322; CARRAZZA, 2004, p. 483).

O tema chegou ao Supremo Tribunal Federal ainda na década de noventa ${ }^{2}$, obrigando aquela Corte a declarar, com nítida razão, a inconstitucionalidade das taxas de iluminação pública, editando, para este fim, a Súmula/Enunciado $n^{\circ} 670^{3}$.

Diante disso, diversos Municípios sofreram com uma severa perda de receitas, e após intensa pressão política por parte das Prefeituras, não restou alternativa ao Poder Constituinte Derivado senão emendar a Constituição da República para incluir em seu corpo um novo tributo, o qual pudesse compensar as perdas decorrentes da declaração de inconstitucionalidade das taxas de iluminação pública por todo o país. Este novo tributo foi acrescido ao texto magno através da Emenda Constitucional $n^{0} 39$ de 2002, a qual se prestou a introduzir o artigo 149-A na Constituição4. Trata-se aqui da atual Contribuição para o financiamento de Serviço de Iluminação Pública (COSIP) ou simplesmente Contribuição de Iluminação Pública (CIP), a qual é, na maioria dos entes da federação, cobrada na fatura de consumo de energia elétrica.

$\mathrm{Na}$ mesma toada, o Supremo Tribunal Federal julgou inconstitucional a chamada Taxa de Limpeza Pública (TLP) cobrada por algumas Prefeituras5, por se tratar de espécie tributária incompatível com a natureza dos serviços de limpeza pública, serviços estes dotados de caráter eminentemente universal e genérico. Para tanto, deuse a edição da Súmula Vinculante $\mathrm{n}^{\circ} 19$, a qual afirma, de maneira categórica, que o único serviço de limpeza urbana que pode vir a ser remunerado por taxa é o serviço de coleta, remoção e tratamento ou destinação de lixo ou resíduos provenientes de imóveis6. Isto porque tais serviços, segundo àquela Corte, ao contrário dos serviços de limpeza pública, podem ser individualizados segundo unidades autônomas de intervenção - residências, comércios, clubes etc.7

Demonstra-se, diante disso, que a evolução jurisprudencial 
do Supremo Tribunal Federal vem caminhando no sentido de se reconhecer a inconstitucionalidade de todas as taxas cobradas em razão da prestação de serviços universais, face a um conflito insuperável entre tais exigências e o disposto no artigo 145, inciso II da Carta Política, o qual diz ser dever das taxas financiar apenas serviços públicos específicos e divisíveis, onde há possibilidade de individualização do beneficiário do serviço.

\section{A questão das taxas de incêndio municipais e a recente posição do Supremo Tribunal Federal}

No ano de 2017 o Supremo Tribunal Federal concluiu o julgamento sobre a inconstitucionalidade da taxa de combate a sinistros instituída e cobrada pelo Município de São Paulo, por meio da Lei Municipal $n^{\circ} 8.822$ de 19788 . Tratava-se de uma taxa criada para fazer face aos custos do Município com os prejuízos decorrentes de sinistros em edificações, além do combate e extinção de incêndios dentro dos limites territoriais da comentada Urbe.

Sucede-se que a Suprema Corte reconheceu a inconstitucionalidade da referida cobrança, baseando-se, para tanto, em dois argumentos:

Primeiramente, com arrimo no art. 144 da Constituição da República, oSupremo reconheceu que as atividades ligadas à prevenção e combate a incêndios são ações desenvolvidas essencialmente pelos Corpos de Bombeiros Militares Estaduais, organizações com previsão constitucional no inciso $\mathrm{V}$ do referido artigo, as quais são ligadas à segurança pública do Estado.

Em segundo lugar, a Suprema Corte afirmou, mais uma vez, que os serviços ligados à segurança pública são serviços de índole incontestavelmente universal, o que inviabiliza sua remuneração através de taxas, sob pena de violação frontal ao disposto no art. 142, inciso II, da Constituição.

Diante disso, o Tribunal Constitucional, no julgamento do Recurso Extraordinário $\mathrm{n}^{\circ}$ 643.247, com repercussão geral, relatado pelo Eminente Ministro Marco Aurélio Mello, julgado em $1^{\circ}$ de agosto de 2017, fixou a seguinte tese:

(...) A segurança pública, presentes a prevenção e o combate a incêndios, faz-se, no campo da atividade precípua, pela unidade da Federação, e, porque serviço essencial, tem como a viabilizá-la a arrecadação de impostos, não cabendo ao Município a criação de taxa para tal fim (...).

Assim, reafirmou a Corte o entendimento de que os serviços 
148 Aspectos históricos e constitucionais da taxa de incêndio do estado do Rio de Janeiro

ligados à segurança pública, notadamente aqueles vinculados à prevenção e combate a incêndios são serviços públicos universais e indivisíveis, não podendo ser remunerados por taxas, mas tão somente pela receita oriunda dos impostos já previstos na competência tributária dos Entes Políticos.

\section{Taxa de incêndio: o caso do Rio de Janeiro}

A taxa de serviços Estaduais, relativa à Prevenção e Extinção de Incêndio foi inicialmente instituída pelo Decreto-Lei no 05 de 1975 (Código Tributário do Estado do Rio de Janeiro) e posteriormente regulamentada pelo Decreto $\mathrm{n}^{\circ} 3.856$ de 1980. Espécie do gênero taxa de serviços, as quais foram estampadas no artigo $2^{\circ}$, inciso II, do Código Tributário do Estado do Rio de Janeiro9, tem como fato gerador os serviços de prevenção e extinção de incêndio, prestados ou colocados à disposição de unidades imobiliárias de utilização residencial ou nãoresidencial, ocupadas ou não, na forma do art. $1^{\circ}$ do Decreto $\mathrm{n}^{\circ} 3.856$ de 1980.

Posteriormente, no ano de 1982, ainda durante o governo de Chagas Freitas, a Lei $n^{\circ} 622$ criou o Fundo Especial do Corpo de Bombeiros do Estado do Rio de Janeiro (FUNESBOM), o qual fora destinado, segundo a referida norma, à:

(...) aplicação de recursos financeiros para reequipamento material, realizações ou serviços, inclusive programas de ensino, de assistência médico-hospitalar e de assistência social, do Corpo de Bombeiros Militar do Estado do Rio de Janeiro, investimentos em equipamentos e projetos de prevenção e combate de incêndios nas cidades e reservas ecológicas, incluindo as áreas da mata atlântica, e manutenção dos órgãos e serviços da Secretaria de Estado de Defesa Civil, voltados prioritariamente para as atividades de capacitação e atualização de recursos humanos, desenvolvimento de programas de valorização e motivação profissional, bem como para o pagamento de despesas de pessoal referentes a gratificações.

Os valores cobrados, fiscalizados e arrecadados pela antiga Secretaria de Estado de Economia e Finanças com a taxa relativa à Prevenção e Extinção de Incêndio, segundo a referida lei e à partir de então, constituiriam receitas pertencentes ao Fundo Especial do Corpo de Bombeiros do Estado do Rio de Janeiro (FUNESBOM), o qual passaria a gerir e aplicar os valores para as finalidades ligadas aos serviços de prevenção e combate a incêndios, inclusive na manutenção 
do material operativo e eventual reequipamento da corporação10.

No ano de 1997, através do Decreto Estadual no 23.695, restou delegado ao Corpo de Bombeiros Militar do Estado do Rio de Janeiro a capacidade tributária ativa em relação à referida taxa, ou seja, aquele órgão deveria então assumir a arrecadação do tributo, inclusive, responsabilizando-se pela manutenção e atualização do cadastro de contribuintes.

Atualmente, a chamada taxa de incêndio é arrecadada pelo Corpo de Bombeiros Militar do Estado do Rio de Janeiro (CBMERJ) e sua aplicação fica a cargo do Fundo Especial do Corpo de Bombeiros (FUNESBOM)11.

\section{Críticas à taxa de incêndio fluminense}

O caráter universal do serviço prestado pelo Corpo de Bombeiros se extrai segundo uma análise topográfico-sistemática da Constituição. $\mathrm{O}$ artigo 144 da Carta, que disciplina as forças de segurança, aduz tratar-se o referido Corpo de serviço ligado à segurança pública, na forma de seu inciso V. Encontra-se o comentado dispositivo no Título $\mathrm{V}$, que trata da Defesa do Estado e das Instituições Democráticas, especificamente no Capítulo III, que versa sobre a segurança pública.

Em relação à taxa de incêndio exigida pelo Corpo de Bombeiros do Estado do Rio de Janeiro (CBMERJ), as principais questões que giram em torno de sua legitimidade caminham no sentido de que se trata de tributo destinado a remunerar serviço público universal, coisa que cabe, notadamente, aos impostos e não às taxas (FALCÃO, 1999 , p. 77). Essa posição converge com o posicionamento doutrinário tradicional acerca das taxas de serviços (NOVELLI, 1992, p. 7).

A questão já foi objeto de elevada controvérsia no âmbito do Tribunal de Justiça do Estado do Rio de Janeiro12, o qual entendeu, contudo, pela constitucionalidade da exação:

NO QUE TANGE À TAXA DE INCÊNDIO, CERTO É QUE SE TRATA DE UM SERVIÇO PÚBLICO DOTADO DAS CARACTERÍSTICAS DE ESPECIFICIDADE E DIVISIBILIDADE. INDIQUE-SE, AINDA, QUE A BASE DE CÁLCULO DIVERGE DAQUELA APRESENTADA PELO IPTU, UMA VEZQUE UMA SE REFERE AO VALOR VENAL DO IMÓVEL E OUTRA À ÁREA CONSTRUÍDA DO IMÓVEL13.

Apesar disto, a tentativa de enquadrar os serviços prestados pelos Corpos de Bombeiros Militares na categoria dos serviços divisíveis não foi das mais felizes. Isto porque, conforme leciona a 
150 Aspectos históricos e constitucionais da taxa de incêndio do estado do Rio de Janeiro

literatura clássica, os serviços de defesa externa, da manutenção da ordem e defesa internas, onde então se insere a segurança pública, são serviços essenciais à existência do Estado enquanto tal (NOVELLI, 1992, p. 7). Objetivam atender às necessidades públicas gerais, donde os beneficiários não podem e nem devem ser individualmente identificados (MEIRELLES, 2005, p. 326; ARAGÃO, 2012, p. 374-375).

Com isso, só se pode concluir que a ausência de referibilidade individual14 prejudica sobremaneira o enquadramento dos serviços prestados pelos Bombeiros na categoria dos serviços divisíveis, como ordena a Constituição em seu art. 145, inciso II. São, ao contrário, exemplo clássico de serviço público ligado à manutenção da ordem interna, o qual, inclusive, é tratado por alguns autores não como serviço público propriamente dito, mas como verdadeira função pública soberana, ao lado de institutos como a diplomacia, a tributação e o poder de polícia administrativa (ARAGÃO, 2012, p. 374).

Admitir que a remuneração dos serviços prestados pelos Corpos de Bombeiros Militares advenha das taxas é conceber que o mesmo tratamento possa ser franqueado a outras corporações de similar natureza, tais como as polícias militares e civis, todos órgãos tratados no Título $\mathrm{V}$ da Constituição da República, título este que versa sobre a Defesa do Estado e das Instituições Democráticas. Essa possibilidade é inconcebível sob a ótica Constitucional.

\section{Apontamentos finais}

O presente estudo serviu-se a analisar a questão da taxa de incêndio exigida no âmbito do Estado do Rio de Janeiro, a qual teve sua arrecadação delegada ao Corpo de Bombeiros Militar do comentado Estado-membro (CBMERJ) e sua guarda e aplicação confiada ao Fundo Especial do Corpo de Bombeiros do Estado do Rio de Janeiro (FUNESBOM). Traçou ainda o delineamento histórico do referido tributo, inclusive, com a evolução jurisprudencial sobre o tema, a qual no âmbito do referido Estado, considerou constitucional a referida exigência.

Fez-nos refletir acerca da natureza jurídica dos serviços prestados pelo Corpo de Bombeiros Militar do Estado do Rio de Janeiro (CBMERJ), concluindo, diante do que se expôs, pela natureza uti universi dos aludidos serviços, o que supõe a inconstitucionalidade da referida exação, tendo em conta que os serviços públicos prestados de maneira indistinta e genérica à toda a população só podem ser remunerados pela receita dos impostos.

Por fim, diante da última decisão do Supremo Tribunal Federal acerca das taxas de incêndio cobradas pelos Municípios, as quais foram consideradas inconstitucionais, restou claro no voto do 
Eminente Ministro Relator - argumento tacitamente admitido pelos demais Membros da Corte - que as taxas cobradas pelos Estados no âmbito de suas atribuições ligadas à segurança pública violam o Texto Magno, notadamente no que se refere às distinções traçadas pelo art. 145, inciso II, da Lei Maior, considerando-se que aqueles serviços são de cunho notadamente universal (uti universi).

\section{NOTAS}

1 Trata-se da divisão pentapartida dos tributos constantes nos artigos 145,148 e 149 da CRFB/88, reafirmada pelo STF quando do julgamento dos seguintes cases: RREE 138.284-8; 146.733 e ADC-1/DF.

2 Precedentes: AI 231132 AgR, Publicações: DJ de 06/08/1999, RTJ 171/1053; RE 231764, Publicações: DJ de 21/05/1999, RTJ 169/742; RE 233332, Publicação: DJ de 14/05/1999.

3Súmula 670: O serviço de iluminação pública não pode ser remunerado mediante taxa.

4 Art. 149-A Os Municípios e o Distrito Federal poderão instituir contribuição, na forma das respectivas leis, para o custeio do serviço de iluminação pública, observado o disposto no art. 150, I e III. (Incluído pela Emenda Constitucional no 39, de 2002)

Parágrafo único. É facultada a cobrança da contribuição a que se refere o caput, na fatura de consumo de energia elétrica. (Incluído pela Emenda Constitucional n ${ }^{\circ}$ 39, de 2002)

5 Cf. “SERVIÇO DE LIMPEZA DE LOGRADOUROS PÚBLICOS E DE COLETA DOMICILIAR DE LIXO. UNIVERSALIDADE. COBRANÇA DE TAXA. IMPOSSIBILIDADE. Tratando-se de taxa vinculada não somente à coleta domiciliar de lixo, mas, também, à limpeza de logradouros públicos, serviço de caráter universal e indivisível, é de se reconhecer a inviabilidade de sua cobrança. Precedentes: RREE 245.539 e 206.777. Recurso extraordinário conhecido e provido." (RE 361.437, Rel. Min. Ellen Gracie, Primeira Turma, DJ 19.12.2002)

6 Súmula Vinculante 19: A taxa cobrada exclusivamente em razão dos serviços públicos de coleta, remoção e tratamento ou destinação de lixo ou resíduos provenientes de imóveis, não viola o artigo 145, II, da 
152 Aspectos históricos e constitucionais da taxa de incêndio do estado do Rio de Janeiro

Constituição Federal.

7 O termo "unidades autônomas de intervenção", estampado no art. 79, inciso II, do CTN, consta do julgamento do Recurso Extraordinário $\mathrm{n}^{\circ}$ 337.349-AgR de relatoria do Eminente Ministro Carlos Velloso, originado da Segunda Turma do STF, assim ementado: "EMENTA: CONSTITUCIONAL. TRIBUTÁRIO. TAXA DE LIMPEZA PÚBLICA: MUNICÍPIO DE BELO HORIZONTE. CF, art. 145, II. CTN, art. 79, II e III. I. - As taxas de serviço devem ter, como fato gerador, serviços públicos específicos e divisíveis, prestados ao contribuinte ou postos a sua disposição. Serviços específicos são aqueles que podem ser destacados em unidades autônomas de intervenção, de utilidade ou de necessidade públicas; e divisíveis, quando suscetíveis de utilização, separadamente, por parte de cada um dos usuários. CTN, art. 79, II e III. II. - Taxa de Limpeza Pública: Município de Belo Horizonte: o seu fato gerador apresenta conteúdo inespecífico e indivisível. III. - Agravo não provido." (Grifamos).

8 Confira-se o disposto no art. $1^{\circ}$ da Lei no 8.822 de 1978, do Município de São Paulo: A Taxa de Combate a Sinistros é devida pela utilização efetiva ou potencial dos serviços municipais de assistência, combate e extinção de incêndios ou de outros sinistros em prédios.

9 “Art. 2. ${ }^{\circ}$ Os Tributos Estaduais são:

I - Imposto sobre:

1. Transmissão Causa Mortis e Doação - ITD;

2. Circulação de Mercadorias e Serviços - ICMS;

3. Propriedade de Veículos Automotores - IPVA;

II - Taxas em razão do exercício do poder de polícia ou pela utilização, efetiva ou potencial, de serviços públicos específicos e divisíveis, prestados ao contribuinte ou postos à sua disposição;

III - Contribuições de melhoria, decorrentes de obras públicas.

\{Redação do Artigo 2. ${ }^{\circ}$, alterado pela Lei Estadual n. ${ }^{\circ}$ 2657/96, vigente desde 01.11.96)."

10 “Art. $2^{\circ}$ - Constituem receitas do FUNESBOM:

(...)

IV - os recursos provenientes da Taxa de Prevenção e Extinção de Incêndio, regulamentada pelo Decreto No 3856, de 29.12.80;" 
11 Disponível em: http:/ / www.funesbom.rj.gov.br

12 Diversos foram os julgados proferidos no âmbito do Tribunal de Justiça do Estado do Rio de Janeiro que reconheceram a constitucionalidade da taxa de incêndio cobrada pelo CBMERJ, com destaque para a Apelação Cível n ${ }^{\circ}$ 2006.001.67382, julgada em 24 abril de 2007, na $1^{\text {a }}$ Câmara Cível e para a Apelação Cível no 2008.001.09619, julgada em 04 de junho de 2008, na $17^{\mathrm{a}}$ Câmara Cível da referida Corte.

13 Trata-se da Apelação Cível no 2007.001.58224, relatada pelo Desembargador Siro Darlan de Oliveira, cujo julgamento ocorreu em 27 de novembro de 2007, na 12 ${ }^{a}$ Câmara Cível do Tribunal de Justiça do Estado do Rio de Janeiro.

14 Segundo aponta Ricardo Lodi Ribeiro, em artigo intitulado O Princípio da Capacidade Contributiva nos Impostos, nas Taxas e nas Contribuições Parafiscais, as taxas são regidas pelo princípio da referibilidade individual, ou seja, deve haver vinculação direta entre aquilo que se paga e aquilo que se recebe como contraprestação estatal específica. Nas palavras do autor: "referibilidade entre a atividade estatal vinculada ao fato gerador e a atuação do contribuinte identificada pelo legislador como núcleo dessa hipótese de incidência". Disponível em: http://www.revistadireito.uerj.br/artigos/OPrincipiodaCapaci dadeContributivanosImpostosnasTaxasenasContribuicoesParafiscais. pdf Acesso em: 31 de agosto de 2018, p. 20-21.

\section{Referências}

ARAGÃO, Alexandre Santos de. Curso de Direito Administrativo. Rio de Janeiro: Forense, 2012.

BALEEIRO, Aliomar. Direito Tributário Brasileiro, atualizada por Misabel Abreu Machado Derzi. Rio de Janeiro: Forense, 2005.

CARRAZZA, Roque Antônio. Curso de Direito Constitucional Tributário. São Paulo: Malheiros, 2004. 
154 Aspectos históricos e constitucionais da taxa de incêndio do estado do Rio de Janeiro

FALCÃO, Amílcar de Araújo. Fato Gerador da Obrigação Tributária. $6^{\mathrm{a}}$ ed. revista e atualizada pelo Prof. Flávio Bauer Novelli. Rio de Janeiro: Forense, 1999.

MEIRELLES, Hely Lopes. Direito Administrativo Brasileiro. 30 edição atualizada por Eurico de Andrade Azevedo [et. al.] São Paulo: Malheiros, 2005.

NOVELLI, Flávio Bauer. Apontamentos sobre o conceito jurídico de taxa. Rio de Janeiro: Revista de Direito Administrativo, $n^{\circ}$ 189, p. 1-38, jul./set., 1992. Disponível em: http://bibliotecadigital.fgv.br/ojs/ index.php/rda/article/view/45279/47706 Acesso em: 31 de agosto de 2018.

ROSA JUNIOR, Luiz Emygdio F. da. Manual de Direito Tributário. Rio de Janeiro: Renovar, 2009.

TORRES, Ricardo Lobo. Curso de Direito Financeiro e Tributário. Rio de Janeiro: Renovar, 1998. 
RDISSERTARDISSERTARDISSERTARDISSHRTARDISSERTARDISSERTARDISL

TARDISSERTARDISSERTARDISSERTARDISSERTARDISSERTARDISSERTARD HRTARDISSERTARDISSERTARDISSERTARDISSERTARDISSERTARDISSERTA] SSERTARDISSERTARDISSERTARDISSERTARDISSERTARDISSERTARDISSER RDISSERTARDISSERTARDISSERTARDISSERTARDISSERTARDISSERTARDISE TARDISSERTARDISSERTARDISSERTARDISSERTARDISSERTARDISSERTARD HRTARDISSERTARDISSERTARDISSERTARDISSERTARDISSERTARDISSERTA] SSERTARDISSERTARDISSERTARDISSERTARDISSERTARDISSERTARDISSER RDISSERTARDISSERTARDISSERTARDISSERTARDISSERTARDISSERTARDISC TARDISSERTARDISSERTARDISSERTARDISSERTARDISSERTARDISSERTARD HRTARDISSERTARDISSERTARDISSERTARDISSERTARDISSERTARDISSERTA] SSERTARDISSERTARDISSERTARDISSERTARDISSERTARDISSERTARDISSER RDISSERTARDISSERTARDISSERTARDISSERTARDISSERTARDISSERTARDISE TARDISSERTARDISSERTARDISSERTARDISSERTARDISSERTARDISSERTARD RTARDISSERTARDISSERTARDISSERTARDISSERTARDISSERTARDISSERTA] SSERTARDISSERTARDISSERTARDISSERTARDISSERTARDISSERTARDISSER RDISSERTARDISSERTARDISSERTARDISSERTARDISSERTARDISSERTARDISE TARDISSERTARDISSERTARDISSERTARDISSERTARDISSERTARDISSERTARD HTARDISSERTARDISSERTARDISSERTARDISSERTARDISSERTARDISSERTA] SSERTARDISSERTARDISSERTARDISSERTARDISSERTARDISSERTARDISSER RDISSERTARDISSERTARDISSERTARDISSERTARDISSERTARDISSERTARDISE TARDISSERTARDISSERTARDISSERTARDISSERTARDISSERTARDISSERTARD HRTARDISSERTARDISSERTARDISSERTARDISSERTARDISSERTARDISSERTA] SSERTARDISSERTARDISSERTARDISSERTARDISSERTARDISSERTARDISSER RDISSERTARDISSERTARDISSERTARDISSERTARDISSERTARDISSERTARDISE TARDISSERTARDISSERTARDISSERTARDISSERTARDISSERTARDISSERTARD HRTARDISSERTARDISSERTARDISSERTARDISSERTARDISSERTARDISSERTA] SSERTARDISSERTARDISSERTARDISSERTARDISSERTARDISSERTARDISSER RDISSERTARDISSERTARDISSERTARDISSERTARDISSERTARDISSERTARDISC 「ARDISSERTARDISSERTARDISSERTARDISSERTARDISSERTARDISSERTARD HRTARDISSERTARDISSERTARDISSERTARDISSERTARDISSERTARDISSERTA] SSERTARDISSERTARDISSERTARDISSERTARDISSERTARDISSERTARDISSER RDISSERTARDISSERTARDISSERTARDISSERTARDISSERTARDISSERTARDISE 「ARDISSERTARDISSERTARDISSERTARDISSERTARDISSERTARDISSERTARD HRTARDISSERTARDISSERTARDISSERTARDISSERTARDISSERTARDISSERTA] SSERTARDISSERTARDISSERTARDISSERTARDISSERTARDISSERTARDISSER RDISSERTARDISSERTARDISSERTARDISSERTARDISSERTARDISSERTARDISE 「ARDISSERTARDISSERTARDISSERTARDISSERTARDISSERTARDISSERTARD HRTARDISSERTARDISSERTARDISSERTARDISSERTARDISSERTARDISSERTA] SSERTARDISSERTARDISSERTARDISSERTARDISSERTARDISSERTARDISSER RDISSERTARDISSERTARDISSERTARDISSERTARDISSERTARDISSERTARDISE TARDISSERTARDISSERTARDISSERTARDISSERTARDISSERTARDISSERTARD HRTARDISSERTARDISSERTARRTARDISSERTARDISSERTARDISSERTARDISS IARDISSERTARDISSERTARDISSERTARDISSERTARDISSERTARRTARDISSER RDISSERTARDISSERTARDISSERTARDISSERTARDISSERTARDISSERTARDISE 
RDISSERTARDISSERTARDISSERTARDISSHRTARDISSERTARDISSERTARDISL

TARDISSERTARDISSERTARDISSERTARDISSERTARDISSERTARDISSERTARD HRTARDISSERTARDISSERTARDISSERTARDISSERTARDISSERTARDISSERTA] SSERTARDISSERTARDISSERTARDISSERTARDISSERTARDISSERTARDISSER RDISSERTARDISSERTARDISSERTARDISSERTARDISSERTARDISSERTARDISE TARDISSERTARDISSERTARDISSERTARDISSERTARDISSERTARDISSERTARD HRTARDISSERTARDISSERTARDISSERTARDISSERTARDISSERTARDISSERTA] SSERTARDISSERTARDISSERTARDISSERTARDISSERTARDISSERTARDISSER RDISSERTARDISSERTARDISSERTARDISSERTARDISSERTARDISSERTARDISC TARDISSERTARDISSERTARDISSERTARDISSERTARDISSERTARDISSERTARD HRTARDISSERTARDISSERTARDISSERTARDISSERTARDISSERTARDISSERTA] SSERTARDISSERTARDISSERTARDISSERTARDISSERTARDISSERTARDISSER RDISSERTARDISSERTARDISSERTARDISSERTARDISSERTARDISSERTARDISE TARDISSERTARDISSERTARDISSERTARDISSERTARDISSERTARDISSERTARD RTARDISSERTARDISSERTARDISSERTARDISSERTARDISSERTARDISSERTA] SSERTARDISSERTARDISSERTARDISSERTARDISSERTARDISSERTARDISSER RDISSERTARDISSERTARDISSERTARDISSERTARDISSERTARDISSERTARDISE TARDISSERTARDISSERTARDISSERTARDISSERTARDISSERTARDISSERTARD HTARDISSERTARDISSERTARDISSERTARDISSERTARDISSERTARDISSERTA] SSERTARDISSERTARDISSERTARDISSERTARDISSERTARDISSERTARDISSER RDISSERTARDISSERTARDISSERTARDISSERTARDISSERTARDISSERTARDISE TARDISSERTARDISSERTARDISSERTARDISSERTARDISSERTARDISSERTARD HRTARDISSERTARDISSERTARDISSERTARDISSERTARDISSERTARDISSERTA] SSERTARDISSERTARDISSERTARDISSERTARDISSERTARDISSERTARDISSER RDISSERTARDISSERTARDISSERTARDISSERTARDISSERTARDISSERTARDISE TARDISSERTARDISSERTARDISSERTARDISSERTARDISSERTARDISSERTARD HRTARDISSERTARDISSERTARDISSERTARDISSERTARDISSERTARDISSERTA] SSERTARDISSERTARDISSERTARDISSERTARDISSERTARDISSERTARDISSER RDISSERTARDISSERTARDISSERTARDISSERTARDISSERTARDISSERTARDISC 「ARDISSERTARDISSERTARDISSERTARDISSERTARDISSERTARDISSERTARD HRTARDISSERTARDISSERTARDISSERTARDISSERTARDISSERTARDISSERTA] SSERTARDISSERTARDISSERTARDISSERTARDISSERTARDISSERTARDISSER RDISSERTARDISSERTARDISSERTARDISSERTARDISSERTARDISSERTARDISE 「ARDISSERTARDISSERTARDISSERTARDISSERTARDISSERTARDISSERTARD HRTARDISSERTARDISSERTARDISSERTARDISSERTARDISSERTARDISSERTA] SSERTARDISSERTARDISSERTARDISSERTARDISSERTARDISSERTARDISSER RDISSERTARDISSERTARDISSERTARDISSERTARDISSERTARDISSERTARDISE 「ARDISSERTARDISSERTARDISSERTARDISSERTARDISSERTARDISSERTARD HRTARDISSERTARDISSERTARDISSERTARDISSERTARDISSERTARDISSERTA] SSERTARDISSERTARDISSERTARDISSERTARDISSERTARDISSERTARDISSER RDISSERTARDISSERTARDISSERTARDISSERTARDISSERTARDISSERTARDISE TARDISSERTARDISSERTARDISSERTARDISSERTARDISSERTARDISSERTARD HRTARDISSERTARDISSERTARRTARDISSERTARDISSERTARDISSERTARDISS IARDISSERTARDISSERTARDISSERTARDISSERTARDISSERTARRTARDISSER RDISSERTARDISSERTARDISSERTARDISSERTARDISSERTARDISSERTARDISE 